\title{
Burnout (síndrome de desgaste profesional) en médicos residentes de anestesiología y su relación con la percepción del clima educacional
}

\author{
Luis A. Neculpán ${ }^{1 *}$ y Martín A. Bruno² \\ ${ }^{1}$ Médico Residente de Anestesiología, Hospital Público Descentralizado Dr. Guillermo Rawson; ${ }^{2}$ Instituto de Ciencias Biomédicas, Facultad de \\ Ciencias Médicas, Universidad Católica de Cuyo, CONICET. San Juan, Argentina
}

\begin{abstract}
Resumen
Introducción: El clima educacional (CE) influye en la motivación de los estudiantes, en la disposición hacia el estudio y en el rendimiento académico. El burnout es una respuesta prolongada a factores estresantes emocionales e interpersonales crónicos en las organizaciones de trabajo. Los médicos residentes son una población de riesgo para el desarrollo de burnout. Objetivos: Evaluar el CE y su relación con el burnout en médicos residentes de anestesiología. Materiales y métodos: El estudio se realizó durante el 2. ${ }^{\circ}$ Encuentro Internacional de Residentes de Anestesiología, organizado por la Federación Argentina de Asociaciones de Analgesia, Anestesia y Reanimación. Se diseñó un estudio analítico de corte transversal. Las variables a analizar fueron: sexo, año de residencia, percepción del CE (cuestionario Postgraduate Hospital Education Environment Measure) y el burnout (cuestionario Maslach Burnout Inventory). Resultados: Respondieron 80 residentes (10\% del total del país). La media del CE fue de 95.5/160 (clima más positivo que negativo, con aspectos a mejorar). Hubo una prevalencia de burnout del 20\%. Existe una asociación estadísticamente significativa entre burnout y una peor percepción del CE (T: 3.51; $p=0.0025)$. Los varones son el grupo más afectado por el burnout $(x 2: 4.88 ; p=0.027)$. Discusión: Los trabajos acerca del CE y el burnout realizados con el mismo instrumento arrojan resultados similares en otras especialidades. Se requieren trabajos prospectivos para evaluar intervenciones que mejoren el CE y el burnout. Conclusiones: Existe una asociación significativa entre el burnout y una peor percepción del CE en los médicos residentes de anestesiología participantes del estudio.
\end{abstract}

Palabras clave: Residencia médica. Clima educacional. Burnout. Educación médica. Profesionalismo.

\section{Burnout (professional burnout syndrome) in anesthesiology medical residents and its relationship with the perception of the educational climate}

\section{Summary}

Introduction: The educational climate (EC) influences the motivation of students, in the readiness to study and in academic performance. Burnout is a prolonged response to emotional and interpersonal chronic stressors in work organizations. The resident doctors are a risk population for burnout development. Objectives: To evaluate the EC and its relationship with 
burnout in medical residents of anesthesiology. Materials and Methods: The study was conducted during the $2^{\text {nd }}$ International Meeting of Residents of Anesthesiology organized by the Argentine Federation of Associations of Analgesia, Anesthesia and Resuscitation. An analytical and cross-sectional study was designed. The variables analyzed were: sex, year of residence, perception of EC (Postgraduate Hospital Education Environment Measurement questionnaire) and burnout (Maslach Burnout Inventory questionnaire). Results: 80 medical residents responded (10\% of the total country). The average of EC was $95.5 / 160$ (climate more positive than negative with aspects to improve). There was a burnout prevalence of $20 \%$. There is a statistically significant association between burnout and a worse perception of $E C(T: 3.51 ; p=0.0025)$. Males are the most affected by burnout $(\times 2: 4.88 ; p=0.027)$. Discussion: These results are similar to the findings of previous studies regarding EC and burnout, whereas same instrument was applied but different medical specialties. Prospective studies are required to further evaluate potential interventions aimed to improve the $E C$ and the burnout. Conclusions: There is a significant association between burnout and a worse perception of the EC within anesthesiology medical resident.

Key words: Medical residency. Educational climate. Burnout. Medical Education. Professionalism.

\section{Introducción}

En los últimos años se destaca en la literatura médica el estudio sobre la respuesta del médico ante situaciones permanentes consideradas estresantes en su ámbito laboral. El burnout es una respuesta prolongada a factores estresantes emocionales e interpersonales crónicos en las organizaciones de trabajo, y se define por tres dimensiones psicológicas: el agotamiento, la despersonalización hacia los demás y el sentimiento de ineficacia ${ }^{1}$. Factores relacionados con la especialidad contribuyen al desarrollo de este síndrome: la constante amenaza de litigio, el tiempo lejos de la familia, la complejidad actual de la medicina moderna, que exige una formación continua constante, y el tiempo laboral confinado a un espacio reducido, entre otros ${ }^{2}$.

El síndrome de desgaste profesional afecta a la salud del trabajador, el profesionalismo, el cuidado de los pacientes y la longevidad dentro de la especialidad ${ }^{3}$.

Este problema es una realidad global ${ }^{4}$. En 2003, Nyssen, et al. publicaron los resultados de un estudio realizado en Bélgica donde observaron los niveles, las causas y los factores asociados con el estrés y el burnout en médicos anestesiólogos, por medio de cuestionarios autoadministrados. Se encontró que el nivel de estrés era similar a la población trabajadora mundial, pero que los médicos anestesiólogos (especialmente aquellos menores de 30 años) tenían altos niveles de burnout en forma de agotamiento emocional ${ }^{5}$.

Los estudios sobre burnout en los anestesiólogos estadounidenses se han concentrado sobre todo en la práctica académica. En 2011, De Oliveira, et al. publicaron dos estudios con altas tasas de respuesta, una investigación de prevalencia en jefes de departamentos académicos de anestesiología y otro en directores de programas de residencia de anestesia. Ellos encontraron que más de la mitad de los jefes de departamento demostraron niveles altos o moderadamente elevados de agotamiento ${ }^{6,7}$.

Sin embargo, el síndrome de desgaste profesional no es algo exclusivo del especialista. Es mucha la evidencia alrededor del mundo que demuestra que el burnout se desarrolla precozmente en la carrera del médico $0^{8,9}$.

Los estudios han demostrado que, aunque los estudiantes de medicina entran en su formación con perfiles de salud mental similares a los de sus pares, grados sustanciales de agotamiento y depresión se hacen evidentes tempranamente durante el proceso de formación médica y aumentan durante la residencia ${ }^{10}$.

El proceso arduo de entrenamiento, durante el cual los médicos residentes tienen poco control sobre su horario, también influye, e impide que los futuros médicos desarrollen las habilidades necesarias para integrar sus responsabilidades personales y profesionales una vez que entran formalmente en la práctica como especialistas. En gran parte de los países anglosajones, el alto costo de la capacitación (en tiempo y dinero) contribuye en los futuros especialistas con una cantidad significativa de la «deuda educativa» que les anima a trabajar largas horas al principio de su carrera, lo que perpetúa el agotamiento adquirido durante la formación ${ }^{11-13}$.

En Argentina se realizó un estudio en los anestesiólogos de la ciudad de Rosario, en el que se encontraron diferencias significativas entre la prevalencia de burnout en médicos especialistas cuando se los comparaba con médicos residentes, y era más elevada en estos últimos ${ }^{14}$.

El clima educacional (CE) de los programas de residencia puede influir en el desempeño del residente, tanto en sus aspectos positivos (como la motivación extrínseca para lograr un aprendizaje significativo) 
como en sus aspectos negativos (relacionados con el síndrome de desgaste profesional). Una forma de evaluar la calidad de los programas de residencia médica es valorar el CE.

El «ambiente educacional» es producto de las relaciones interpersonales, del estilo de comunicación dominante, de las prácticas habituales (usos y costumbres), de las presiones o factores estresantes (sistema de reconocimiento $\mathrm{y} / \mathrm{o}$ sanciones), de los valores $\mathrm{y}$ principios. El ambiente influye en la motivación de los estudiantes, en la disposición hacia el estudio y en el rendimiento académico. Es decir, tiene una influencia en los resultados, la satisfacción y el éxito de los estudiantes $^{15-17}$.

Genn, et al. destacan la extraordinaria complejidad del ambiente en la educación médica. Subrayan que si se quiere describir o evaluar el currículo de una escuela de medicina es necesario considerar el ambiente organizacional que define el $\mathrm{CE}^{16,17}$.

Como refiere Hutchinson, en la teoría de aprendizaje del adulto tiene tanta importancia el contexto y el clima como la formación educacional ofrecida y la experiencia compartida. La motivación extrínseca desempeña un papel especial para lograr un aprendizaje significativo ${ }^{18}$.

A nivel internacional, de Oliveira, et al. estudiaron en Brasil la relación entre calidad de vida, CE y estrategias de aprendizaje en residentes de anestesiología. Este estudio no pudo detectar ninguna asociación significativa entre el rendimiento académico y las percepciones de los residentes acerca de su calidad de vida subjetiva o sobre el medio ambiente educativo de sus residencias ${ }^{19}$.

En Argentina existen investigaciones del CE en programas de residencia. Llera, et al. correlacionaron CE y burnout en 6 programas de residencia, de los cuales solo una especialidad crítica se encontraba dentro de la muestra (terapia intensiva), en el contexto de un hospital privado universitario ${ }^{20}$. Galli, et al. analizaron el CE en residentes de la Ciudad Autónoma de Buenos Aires sin correlacionarlo con burnout, y tampoco incluyeron en su muestra residentes de anestesiología ${ }^{21}$. El mismo grupo evaluó el CE en residentes de cardiología de hospitales públicos y privados 22 .

Después de relevar la bibliografía no se han encontraron estudios sobre la correlación entre el CE y el burnout, realizados con instrumentos validados, en el contexto de los programas de residencia de anestesiología en Argentina, como así tampoco a nivel internacional. Smith, et al. ${ }^{23}$ publicaron el desarrollo de un cuestionario de CE en Australia, pero dicho instrumento no ha sido validado internacionalmente aún.

\section{Materiales y métodos}

El estudio se realizó en el contexto del 2.ํㅡㄹ Encuentro Internacional de Residentes de Anestesiología, organizado por la Federación Argentina de Asociaciones de Analgesia, Anestesia y Reanimación, que se llevó a cabo en la provincia de Córdoba en el mes de marzo de 2017. Se invitó a participar a todos los residentes que estuvieron presentes, incluso a los residentes extranjeros. Se diseñó un estudio observacional, analítico y de corte transversal.

Las variables a analizar fueron: sexo, año de residencia (1, 2, 3 o 4), percepción del CE y el burnout.

Para la medición del CE se utilizó el cuestionario Postgraduate Hospital Education Environment Measure (PHEEM). Este instrumento creado por Roff, et al. ${ }^{24}$ cuenta con 40 ítems operacionalizados con una escala de Likert de 0 a 4. Evalúa tres dominios: percepción del rol de autonomía (puntaje de 0-56), percepción de la enseñanza (puntaje de 0-60) y percepción del respaldo social (puntaje 0-44). La suma total del cuestionario es de 160 puntos.

Para la evaluación del burnout se utilizó el Maslach Burnout Inventory Human Service Survey (MBI), que cuenta con 22 ítems, con un puntaje en una escala de Likert entre 0 y $6^{25}$. Este instrumento, ampliamente utilizado, incluye 22 ítems que investigan las 3 dimensiones mensurables de burnout: agotamiento emocional (9 preguntas), despersonalización (5 preguntas) y realización personal (8 preguntas).

El resultado principal fue la asociación entre CE y cada subescala del MBI. Se aceptó la medición del CE como variable continua, a fin de hacer comparable el análisis de nuestros resultados con la literatura publicada.

Se analizó la correlación entre las tres subescalas del MBI y las tres subescalas del PHEEM.

Se analizaron los datos de evaluación del CE y el burnout en forma general y según las variables de sexo y año de residencia.

Para el análisis estadístico de cada dimensión del $\mathrm{MBI}$ se dividieron los rangos de las puntuaciones en tres categorías: bajo (agotamiento emocional $\leq 18$, despersonalización $\leq 5$, realización personal $\geq 40$ ), medio (agotamiento emocional 19-26, despersonalización $\leq$ 6-9, realización personal 34-39) y alto (agotamiento emocional $\geq 27$, despersonalización $\geq 10$, realización personal $\leq 33$ ). Se analizó la presencia del síndrome, considerando burnout cuando el participante tuviese valores altos en las tres dimensiones, o bien en dos de ellas valores altos y, en la tercera, moderados. 
Posteriormente se compararon las medias de resultado global de evaluación del CE entre los residentes con burnout y sin él.

\section{Tamaño muestral}

Se calculó un tamaño muestral de 77 encuestados, para un coeficiente de correlación ( $r$ ) esperado de 0.45 (de acuerdo a otros trabajos similares), con un poder del $95 \%$ y un nivel de confianza del $95 \%$ y previendo una pérdida de datos del $10 \%$.

\section{Análisis estadístico}

Se determinó la normalidad de los datos de CE mediante la prueba de Smirnov-Kolmogorov. Los datos que presentaron distribución normal (paramétricas) se analizaron con la prueba T-test y Anova. Para el análisis de datos categóricos (no paramétricos) se utilizó la prueba de chi-cuadrado $\left(\mathrm{X}^{2}\right)$, la prueba exacta de Fisher y el coeficiente se Spearman ( $r$ ). Se tomó como significativo un valor de $p$ menor de 0.05 . Los datos fueron analizados con Excel y GraphPad Prism.

\section{Aspectos éticos}

Ambos cuestionarios fueron autoadministrados, anónimos y de respuesta voluntaria. Se aseguró la confidencialidad de los datos. Se otorgó el cuestionario a los residentes presentes en el 2. Encuentro Internacional de Residentes de Anestesiología.

El trabajo fue evaluado y aprobado para su realización por el Comité de Docencia e Investigación del Hospital Público Descentralizado Dr. Guillermo Rawson (San Juan, Argentina) y por el Comité Ejecutivo de la Federación Argentina de Asociaciones de Analgesia, Anestesia y Reanimación.

\section{Resultados}

En total respondieron 80 residentes (edad promedio: 29 años 27-32), que representan el 10\% del total del país, de 15 programas diferentes de residencias de Argentina. Se excluyeron del análisis los residentes de otros países que asistieron al encuentro. La población evaluada se describe en la tabla 1.

La evaluación del CE obtuvo una media de clima total de 95.5/160 con una desviación estándar de 21.8. Esta medida del CE expresa un clima más positivo que negativo.
Tabla 1. Población de residentes encuestados

\begin{tabular}{|l|c|}
\hline & Total (\%) \\
\hline Año de residencia & \\
Primero & $26(32.5)$ \\
Segundo & $24(30)$ \\
Tercero & $18(22.5)$ \\
Cuarto & $12(15)$ \\
\hline Sexo & \\
Masculino & $51(63.75)$ \\
Femenino & $29(36.25)$ \\
\hline Beca & \\
Públicas & $74(92.5)$ \\
Privadas & $6(7.5)$ \\
\hline
\end{tabular}

La evaluación de las subescalas del PHEEM se muestra en la tabla 2.

En las figuras 1-4 se se muestra la distribución de respuestas de CE total, de la dimensión de autonomía, la dimensión de respaldo social y la dimensión de calidad de la enseñanza.

En un análisis más detallado podemos observar que la dimensión respaldo social fue la mejor evaluada por la mayoría de los médicos residentes (Fig. 3). En particular, el ítem con mayor puntaje de respuesta fue «Tengo buena colaboración con otros residentes de mi nivel» (media: 3.275/4) y «El entrenamiento en esta rotación clínica me hace sentir preparado» (media: 3.075/4). En cuanto a los aspectos a mejorar, podemos observar que el $21 \%$ de los residentes estudiados percibe el CE con un puntaje menor a 80/160. Este resultado corresponde con una percepción del CE como «lleno de problemas». El análisis de la dimensión «Calidad de la enseñanza» desprende que el $30 \%$ de los residentes percibe a la enseñanza como de «escasa calidad y con falta de oportunidades de aprendizaje».

No hubo diferencias en la percepción del CE entre los médicos residentes de distintos años (Anova F: $0.85 ; p=0.46$ ) y tampoco hubo diferencias acerca de la percepción del CE entre varones y mujeres ( $\mathrm{T}: 0.04 ; p=0.63$ ).

Los resultados de la evaluación del burnout se muestran en la tabla 3. Se analizaron las diferencias de cada subescala del MBI por sexo y año de residencia. El análisis con Anova de las subescalas del MBI para los diferentes años de residencia no arrojo diferencias estadísticamente significativas entre los 4 grupos. El análisis con $T$ de Student para cada subescala del MBI entre varones y mujeres solo arrojo diferencias estadísticamente significativas en la subescala de despersonalización ( $\mathrm{T}: 3.10 ; p=0.002)$, y fueron los médicos 
Tabla 2. Evaluación del clima educacional (Posgraduate Hospital Educational Environment Measure)

\begin{tabular}{|l|c|c|c|c|}
\hline & Media & Desviación estándar & Mediana & Rango intercuartil (25-75) \\
\hline Clima total & 95.5 & 21.80 & 97.5 & $83.5-109.5$ \\
\hline Autonomía & 33.31 & 8.09 & 34.0 & $28-39$ \\
\hline Respaldo social & 27.16 & 6.03 & 28 & $24-31$ \\
\hline Enseñanza & 35.02 & 9.44 & 36 & $29-41$ \\
\hline
\end{tabular}

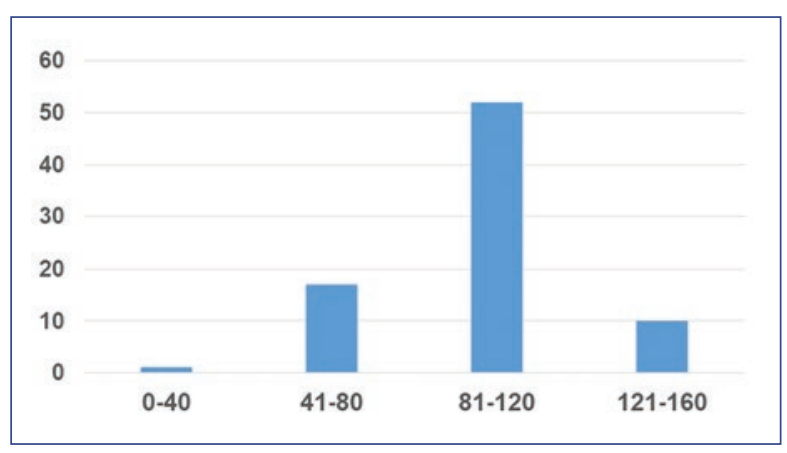

Figura 1. Distribución de respuestas de clima educacional total. Eje de las ordenadas: número de residentes. Eje de abscisas: rangos de respuestas. Rango de respuestas: 0-40, clima educacional muy pobre; 41-80, clima educacional Ileno de problemas; 81-120, clima educacional más positivo que negativo; 121-160, clima educacional excelente.

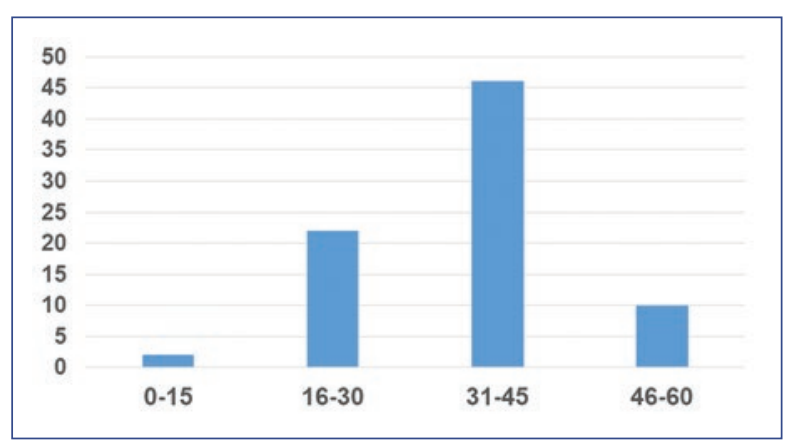

Figura 2. Distribución de respuestas de la dimensión calidad de la enseñanza. Eje de las ordenadas: número de residentes. Eje de abscisas: rangos de respuestas. Rango de respuestas: $0-15$, calidad de la enseñanza muy pobre; 16-30, calidad de la enseñanza que necesita revisión; 3145, calidad de la enseñanza en buena dirección; 46-60, calidad de la enseñanza con maestros modelos.

residentes varones los que tuvieron valores más altos de despersonalización.

De acuerdo a la definición adoptada según nuestro protocolo tomando en cuenta las 3 subescalas, 16

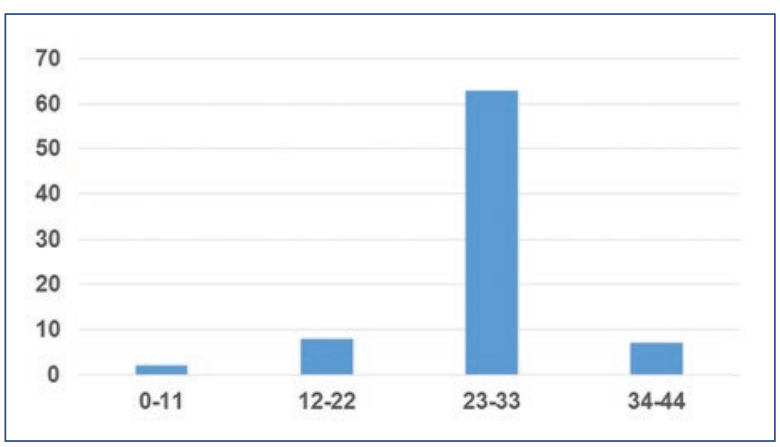

Figura 3. Distribución de respuestas de la dimensión respaldo social. Eje de las ordenadas: número de residentes. Eje de abscisas: rangos de respuestas. Rango de respuestas: $0-11$, no existe respaldo social; $12-22$, el ambiente educacional no es placentero; 23-33, respaldo social con más pros que contras; 34-44, ambiente educacional con buen respaldo social.

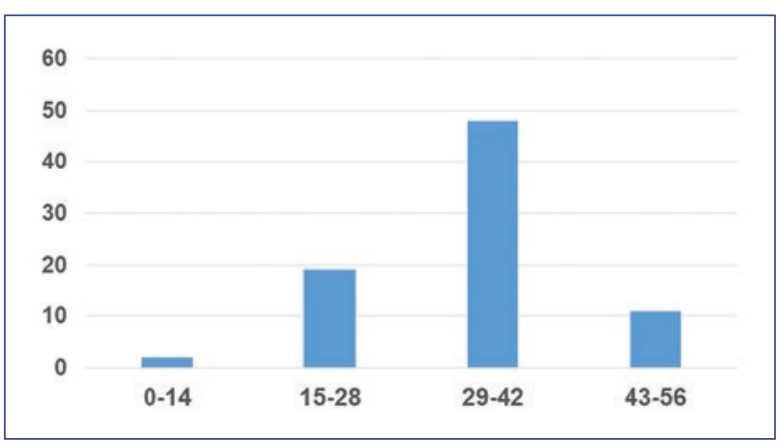

Figura 4. Distribución de respuestas de la dimensión autonomía. Eje de las ordenadas: número de residentes. Eje de abscisas: rangos de respuestas. Rango de respuestas: $0-14$, autonomía muy pobre; 15-28, autonomía negativa; 29-42, autonomía positiva; 43-56, autonomía excelente.

encuestados (20\%) presentaban valores compatibles con el síndrome.

Luego de dividir la muestra según la presencia o la ausencia de burnout, las dos poblaciones mostraron 
Tabla 3. Resultados del Maslach Burnout Inventory

\begin{tabular}{|l|c|c|c|c|}
\hline & Media & Desviación estándar & Mediana & Rango intercuartil (25-75) \\
\hline Agotamiento & 22.14 & 10.35 & 21 & $14-30$ \\
\hline Despersonalización & 7.85 & 5.49 & 7 & $4-11$ \\
\hline Realización & 37.91 & 6.40 & 39 & $35-43$ \\
\hline
\end{tabular}

Tabla 4. Población según la presencia de burnout

\begin{tabular}{|c|c|c|c|}
\hline & Burnout sí = 16 (20\%) & Burnout no $=64(80 \%)$ & Significación $\mathrm{p}<0.05$ \\
\hline $\begin{array}{c}\text { Año de } \\
1 \\
2 \\
3 \\
4\end{array}$ & $\begin{array}{l}7 \\
4 \\
4 \\
1\end{array}$ & $\begin{array}{l}19 \\
20 \\
14 \\
11\end{array}$ & $p=0.56$ \\
\hline $\begin{array}{l}\text { Sexo } \\
\text { Varón } \\
\text { Mujer }\end{array}$ & $\begin{array}{c}14 \\
2\end{array}$ & $\begin{array}{l}37 \\
27\end{array}$ & $p=0.027$ \\
\hline Clima & $75.75(49.08-102.42)$ & $100.43(83.03-117.83)$ & $p=0.0025$ \\
\hline
\end{tabular}

Tabla 5. Correlación entre clima total y subescalas del Posgraduate Hospital Educational Environment Measure (autonomía, respaldo social y enseñanza), con las tres subescalas del Maslach Burnout Inventory (agotamiento, despersonalización y realización). Se destacan en negrita las correlaciones que muestran significación estadístic

\begin{tabular}{|l|c|c|c|}
\hline & Agotamiento & Despersonalización & Realización \\
\hline Clima total & $-0.4081(\mathbf{p}=\mathbf{0 . 0 0 0 2})$ & $-0.2191(p=0.0508)$ & $0.4172(\mathbf{p}=\mathbf{0 . 0 0 0 1 )}$ \\
\hline Enseñanza & $-0.2318(\mathbf{p}=\mathbf{0 . 0 3 8 6})$ & $-0.1566(p=0.1655)$ & $0.3235 ;(\mathbf{p}=\mathbf{0 . 0 0 3 4 )}$ \\
\hline Respaldo social & $-0.4329(\mathbf{p}<\mathbf{0 . 0 0 0 1 )}$ & $0.1147(p=0.3109)$ & $0.4592(\mathbf{p}<\mathbf{0 . 0 0 1 )}$ \\
\hline Autonomía & $-0.4974(\mathbf{p}<\mathbf{0 . 0 0 0 1 )}$ & $-0.3104(\mathbf{p}=\mathbf{0 . 0 0 5 1})$ & $0.4341(\mathbf{p}<\mathbf{0 . 0 0 0 1 )}$ \\
\hline
\end{tabular}

diferencias significativas en cuanto a la evaluación del CE y el sexo, y son los varones los que predominan en la población con burnout (Tabla 4).

Al evaluar la correlación entre CE total con cada una de las subescalas del MBI se encontró una correlación negativa significativa entre clima total con agotamiento y una correlación positiva significativa entre clima total y realización personal.

Al evaluar las subescalas respectivas de ambos instrumentos, la subescala agotamiento presentó una correlación negativa significativa con las tres dimensiones del PHEEM (calidad de la enseñanza, respaldo social y autonomía). La subescala despersonalización mostró una correlación negativa significativa solo con la dimensión autonomía. Finalmente, la subescala realización personal mostró una correlación positiva significativa con las tres dimensiones del PHEEM (Tabla 5).
La aplicación del PHEEM no presento dificultades en la comprensión de los ítems. Mostró una adecuada confiabilidad con un coeficiente alfa de Chronbach de 0.928 .

\section{Discusión}

Este estudio pone de manifiesto la relación entre el síndrome de desgaste profesional y la percepción del CE en médicos residentes de anestesiología.

La formación en anestesiología debe ser interpretada como un proceso complejo, que atraviesa las individualidades y los aspectos estrictamente relacionados con la adquisición de competencias y habilidades ${ }^{26}$. Es por esto que los estudios acerca del CE proveen información desde otra perspectiva para la toma de decisiones en la formación de posgrado de los futuros especialistas en anestesiología. 
La percepción que poseen los médicos residentes participantes acerca del CE es «más positiva que negativa, con aspectos a mejorar». Los trabajos acerca del CE realizados con el mismo instrumento arrojan resultados similares en otras especialidades. Wall, at al. informan valores de CE similares en varios países: Brasil (95), Chile (98), Holanda (105), Reino Unido (112, unidades de cuidados intensivos), Reino Unido (113, foundation programs) ${ }^{27}$. Luego de una revisión sistemática sobre los instrumentos para evaluar el CE, Soemantri, et al. concluyen que el PHEEM es el instrumento más adecuado para evaluar programas de residencia ${ }^{15}$. Este instrumento ha sido traducido, adaptado y validado a múltiples idiomas. Sin embargo, debemos señalar el aporte que realizan Castanelli, et al. ${ }^{28}$, que en 2017 , luego de que nuestro grupo haya iniciado este trabajo, publicaron un nuevo instrumento utilizado en médicos residentes de anestesiología australianos. Es probable que este instrumento sea más adecuado para la evaluación del CE en programas de anestesiología. Futuros trabajos en el área podrían ser la traducción, la adaptación y la validación transcultural de dicho instrumento. Los resultados que obtienen Castanelli, et al. se hacen incomparables con los obtenidos en el presente trabajo debido a que las dimensiones de su cuestionario son distintas.

Por otro lado, los médicos anestesiólogos están expuestos a varios factores de riesgo para desarrollar estrés y burnout, debido a su posición en la primera línea de atención al paciente ${ }^{4}$. En nuestro trabajo se encontró una prevalencia de burnout del $20 \%$, aproximadamente. Este resultado se asemeja mucho al trabajo de Llera, et al. ${ }^{19}$, realizado en médicos residentes de varias especialidades. El trabajo de Montenegro, et. al. ${ }^{13}$ caracteriza el síndrome de desgaste profesional de acuerdo al grado profesional y al género en anestesiólogos. Dentro de sus resultados, los niveles de burnout son más altos en médicos residentes que en especialistas, y en médicos residentes supera la prevalencia del $50 \%$. En cuanto al género, el presente trabajo arroja diferencias significativas en cuanto a la prevalencia de burnout entre mujeres y varones, y los varones son los más afectados. Montenegro, et al..$^{13}$ obtienen prevalencias de burnout estadísticamente similares en ambos géneros.

Se encontraron diferencias estadísticamente significativas en la percepción del CE entre médicos residentes con burnout y sin burnout, y los médicos residentes con burnout fueron los que tuvieron una peor percepción del CE. Este resultado coincide con el trabajo de Llera, et al. ${ }^{19}$ y el de Goñi, et al. ${ }^{29}$ En 2017, Castanelli, et al. publicaron un estudio de similares características en la población australiana, pero utilizando otro cuestionario para evaluar el $\mathrm{CE}^{30}$. Ellos encontraron una correlación significativa entre burnout y una peor percepción del CE.

En cuanto a las limitaciones del presente trabajo, y debido al diseño del estudio, solo se puede establecer una asociación entre burnout y una peor percepción del CE, sin poder arribar a una conclusión acerca de causalidad. ¿Es el CE el que provee las condiciones para que los médicos residentes tengan burnout o es al revés? Nosotros coincidimos con la discusión de Goñi, et al. ${ }^{23} y$ de Castanelli, et al.$^{30}$, en que las condiciones de formación son un componente causal más (no el único, ni el más importante) dentro de los factores estresantes que tienen como consecuencia el desarrollo de burnout y que, por ende, es probable que al modificar dichas condiciones se pueda disminuir la prevalencia de burnout. Otra limitación que observamos del presente trabajo es que no se pueden realizar análisis específicos por programas.

El estudio efectuado representa una de las pocas investigaciones que analizan la relación entre el síndrome de desgaste profesional con los aspectos formativos-educacionales en médicos residentes de anestesiología.

Como posibles estudios a ampliar se pueden mencionar la posibilidad de realizar una comparación entre programas de residencia ligados a universidades y aquellos que no, y el posible efecto que tengan la adquisición de programas formales universitarios. Otro trabajo futuro podría utilizar dichos cuestionarios para la evaluación de intervenciones en educación de posgrado de médicos residentes de anestesiología a través de estudios prospectivos.

\section{Conclusión}

Los residentes de anestesiología participantes del estudio percibieron al CE como más positivo que negativo, con aspectos a mejorar. Existe una prevalencia de burnout del $20 \%$ y dicha condición es más importante en médicos residentes varones. Existe una asociación significativa entre el burnout y una peor percepción del CE.

\section{Agradecimientos}

Agradecemos a la Asociación Sanjuanina de Analgesia, Anestesia y Reanimación su colaboración para hacer factible esta investigación. En particular al Dr. Marcelo Casanova y al Dr. Enrique Zorrilla por 
confiar en nuestra propuesta de investigación y proveer la colaboración necesaria para poder llevarla a cabo.

\section{Conflicto de Intereses}

Los autores declaran no poseer conflicto de intereses.

\section{Financiamiento}

Los autores no han recibido financiación para realizar esta investigación.

\section{Bibliografía}

1. Maslach C, Shaufeli W, Leiter M. Job burnout. Annu Rev Psychol. 2001 52:397-422.

2. Vinson A. Burnout in anhestesiologist: an update. ASA Monitor. 2014; 78(11):18-20

3. Paladino M, Martinetti H. La salud del anestesiólogo (primera parte) Factores de agresión y la respuesta orgánica. Rev Arg Anest. 2005; 63(1):45-59.

4. Rama-Maceiras $P$, Jokinen J, Kranke $P$. Stress and burnout in anesthesia: a real world problem? Curr Opin Anesthesiol. 2015;28:151-8.

5. Nyssen A, Hansez I, Baele P, Lamy M, De Keyser V. Occupational stress and burnout in anaesthesia. Br J Anaesth. 2003;90(3):333-7.

6. De Oliveira GS, Ahmad S, Stock M, Harter RL, Almeida MD, Fitzgerald PC et al. High incidence of burnout in academic chairpersons of anesthesiology: should we be taking better care of our leaders? Anesthesiology 2011;114(1):181-93.

7. De Oliveira GS, Almeida M, Ahmad S, Fitzgerald PC, McCarthy RJ. Anesthesiology residency program director burnout. J Clin Anesth. 2011 23(3):176-82.

8. Shanafelt T. Burnout in anesthesiology: a call to action. Anesthesiology. 2011;114:1-2.

9. Ishak W, Lederer S, Mandili C, Nikravesh R, Seligman L, Vasa M, et al. Burnout during residency training: a literature review. J Grad Med Educ. 2009;1(2):236-42.

10. Dyrbye L, Thomas M, Shanafelt T. Systematic review of depression, anxiety, and other indicators of psychological distress among U.S. and canadian medical students. Acad Med. 2006;81(4):354-73.
11. Goiten L, Shanafelt T, Nathens A, Curtis JR. Effects of resident work hour limitations on faculty professional lives. J Gen Intern Med. 2008; 23(7):1077-83

12. Thomas N. Resident burnout. JAMA. 2004;292(23):2880-9.

13. Chan A. Burnout in residents. ASA Monitor. 2014;78(4):54-5.

14. Montenegro S, Elena G, Tarres M. Burnout en anestesiólogos. Relación con el género y el grado profesional. Rev Arg Anest. 2013;71(3):8-17.

15. Soemantri $D$, Herrera $C$, Riquelme $A$. Measuring the educational environment in health professions studies: a systematic review. Med Teach. 2010;32:947-52.

16. Genn JM. AMEE Medical Education Guide No. 23 (Part1): Curriculum, environment, climate, quality and change in medical education-a unifying perspective. Med Teach. 2001;23(4):337-44

17. Genn JM. AMEE Medical Education Guide No. 23 (Part 2): Curriculum, environment, climate, quality and change in medical education-a unifying perspective. Med Teach. 2001;23(5):445-54.

18. Hutchinson L. Educational environment. BMJ. 2003;326:810-2.

19. De Oliveira Filho G, Vieira JE. The relationship of learning environment, quality of life, and study strategies measures to anaesthesiology resident academic performance. Anesth Analg. 2007;104:1467-72.

20. Llera J, Durante E. Correlación entre el clima educacional y el síndrome de desgaste profesional en los programas de residencia de un hospital universitario. Arch Arg Pediatr. 2014;112(1):6-11.

21. Galli A, Brissón ME, Soler C, Yulitta H, Eiguchi K. Evaluación del ambiente educacional en sistemas de residencias médicas del Gobierno de la Ciudad Autónoma de Buenos Aires. Rev Arg Educ Med. 2015;6(3):100-6.

22. Galli A, Brissón ME, Soler C, Lapresa S, Alves de Lima A. Evaluación del ambiente educacional en residencias de cardiología. Rev Argent Cardiol. 2014;82:396-401.

23. Smith N, Castanelli D. Measuring the clinical learning environment in anaesthesia. Anaesth Intensive Care. 2015;43:199-203.

24. Roff S, McAleer S, Skinner A. Development and validation of an instrument to measure the posgraduate clinical learning and teaching educational environment for hospital based-junior doctors in the UK. Med Teach. 2005;27(4):326-31.

25. Maslach C, Jackson S, Leiter M. Maslach Burnout Inventory. $3^{\text {rd }}$ ed. Palo Alto, CA: Consulting Psychologists Press; 1996.

26. Saez-Fernandez A, Sistac-Ballarin JM. Docencia y Formación. Un camino de futuro en el desarrollo de la Anestesiología y Reanimación del Siglo XXI. Rev Esp Anestesiol Reanim. 2009;56:401-2.

27. Wall D, Clapham M, Riquelme A, Veira J, Cartmill R, Aspegren K, et al. Is PHEEM a multi-dimensional instrument? An international perspective. Medical Teacher. 2009:31:521-7.

28. Castanelli $D$, Smith N. Measuring the anaesthesia clinical learning environment at the department leve is feasible and reliable. BJA. 2017; 118 (5):733-9.

29. Goñi M, Danza A, Urgoiti M, Durante E. Correlación entre clima educativo y síndrome de burnout en practicantes internos de la carrera de Doctor en Medicina de Uruguay. Rev Méd Urug. 2015;31(4):272-81.

30. Castanelli D, Wickramaarachchi S, Wallis S. Burnout and the learning environment of anaesthetic trainees. Anaesth Intensive Care. 2017;45(6):744-51. 